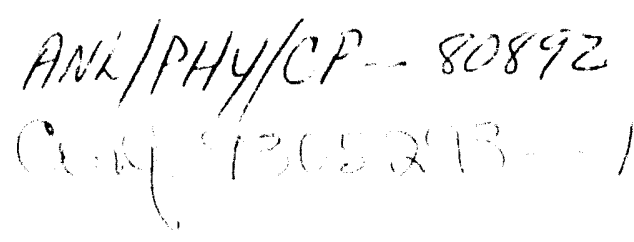

\title{
Nuclear structure studies with intermediate energy probes
}

\author{
T.-S. H. Lee
}

Physics Division, Argonne National Laboratory, Argonne, Ilinois 60439-4843 USA

\begin{abstract}
Nuclear structure studies with pions are reviewed. Results from a recent study of $1 \mathrm{p}$-shell nuclei using $\left(e, e^{\prime}\right),\left(\pi, \pi^{\prime}\right)$ and $(\gamma, \pi)$ reactions are reported. Future nuclear structure studies with $\mathrm{GeV}$ electrons at CEBAF are also briefly discussed.
\end{abstract}

The study of nuclear structure with intermediate energy probes has been one of the major research topics since the time that the meson facilities at LAMPF, TRIUMF, and PSI started to produce high quality pion and proton beams more than 20 years ago. With the new electron facilities at MIT-Bates, Mainz, NIKHEF, and CEBAF, this research is expected to be further pursued in the next decade. In this talk, I will first summarize the main advances in the studies using pions, and then report on the results from a recent study of 1 p-shell nuclei using (e,e'), $\left(\pi, \pi^{\prime}\right)$, and $(\gamma, \pi)$ reactions. Finally, I will discuss the new opportunities at CEBAF.

At intermediate energies, the $\pi$-nucleus interaction is dominated by the formation of the $\Delta$ resonance in the $\mathrm{P}_{33}$ channel of $\pi \mathrm{N}$ scattering. The dominant $\pi \mathrm{N}$ scattering operator can be written as the following form

$$
t(E)=h_{\pi N} \leftarrow \Delta \frac{1}{E-E_{R}+\frac{i}{2} \Gamma_{f}} h_{\Delta \rightarrow \pi N}
$$

where the vertex interaction $h_{\Delta \leftrightarrow \pi N}$ describes the formation and the decay of the $\Delta$ in $\pi \mathrm{N}$ collisions. The positicn and the width of the $\Delta$ are known to be $E_{R} \sim 180 \mathrm{MeV}$ and $\Gamma_{f} \sim 120$ $\mathrm{MeV}$. By fitting the $\pi \mathrm{N}$ phase shifts in $\mathrm{P}_{33}$ channel, the vertex interaction $h_{\Delta \leftrightarrow \pi N}$ can be determined phenomenologically. It is obvious that the vertex interaction $h_{\Delta \leftrightarrow \pi N}$ can generate pion (or $\Delta$ ) multiple scattering from the nucleons inside the nucleus. Another important pionnucleus interaction mechanism is the absorption (annihilation) of the $\Delta$ by other nucleons. The simplest absorption mechanism is due to the one-pion-exchange $\Delta \mathrm{N} \leftrightarrow \mathrm{NN}$ transition. Because of these many-nucleon effects, $\Delta$-propagation in nuclear medium is different from the simple Briet-Wigner form of Eq. (1). The $\pi \mathrm{N}$ scattering operator inside the nucleus is of the following form 


$$
\tau(E)=h_{\pi N} \leftarrow \frac{1}{E-E_{R}+\frac{i}{2} \Gamma f^{-\Sigma^{s . c .}}(E)-V_{\Delta}(E)} h_{\Delta \rightarrow \pi N},
$$

where $\Sigma^{s . c}$.(E) contains the effects due to the multiple scattering processes, $V_{\Delta}(E)$ contains the effects due to the absorption of the $\Delta$ by nuclear medium.

The medium effect on the $\Delta$-propagation has been the focus of very extensive theoretical efforts. The most fruitful approach so far is to determine it within the $\Delta$-hole model [1] of pion-nucleus scattering. In this model, the multiple scattering part $\Sigma^{\mathrm{s} . c}$.(E) is calculated from the elementary $\pi N$ scattering amplitude. The $\Delta$-nucleus potential $V_{\Delta}(E)$ is then determined phenomenologically by fitting the pion-nucleus scattering data. Qualitatively, it was found that $\operatorname{Im}\left(V_{\Delta}\right) \sim(-40) \rho(r) \mathrm{MeV}$ for $\mathrm{A} \leq 16$ nuclei, where $\rho(r)$ is the nuclear density. However, very limited work on the $\Delta$-hole model for heavier nuclei has been done. The A-dependence of $V_{\Delta}$ is therefore not very well known. A more ambitious approach [2] is to calculate $V_{\Delta}(E)$ from a many-body Hamiltonian with $\mathrm{N}, \pi$, and $\Delta$ degrees of freedom. However, only very qualitative results have been obtained since the starting Hamiltonian has not been able to describe quantitatively the $\pi$ and $\Delta$ dynamics in the elementary $N N$ and $\pi \mathrm{d}$ reactions. At the present time, the information extracted from the $\Delta$-hole model is most useful in practice.

Once the medium-corrected $\pi \mathrm{N}$ scattering operator $\tau(\mathrm{E})$, Eq. (2) is determined, the pionnucleus scattering can be described by the well-developed multiple scattering theories. The most widely used formulation was developed by Kerman, McManus and Thaler [3]. In this approach, the pion-nucleus elastic scattering operator is governed by the following LippmanSchwinger equation

$$
T_{00}(E)=U_{00}(E)+U_{00}(E) \frac{1}{E-H_{0}+i \varepsilon} T_{00}(E)
$$

where $U_{00}(E)$ is an optical potential. The inelastic scattering is then calculated by using the Distorted-Wave-Impulse-Approximation (DWIA)

$$
T_{f 0}=\left\langle\chi_{f}^{(-)}\left|U_{f 0}(E)\right| \chi_{0}^{(+)}\right\rangle
$$

where the distorted waves $\chi^{ \pm}$are generated from the ortical potential by solving Eq. (3). The important feature of this multiple scattering formulation is that the pion-nucleus potentials $U_{00}$ and $U_{\mathrm{f} 0}$ are microscopically calculated from the nuclear wave functions and the mediumcorrected $\pi \mathrm{N}$ amplitude $\tau(\mathrm{E})$. In the first-order approximation, we have

$$
U_{f 0}(E)=\left\langle\Phi_{f}|\tau(E)| \Phi_{0}\right\rangle
$$


If the medium-corrected $\tau(\mathrm{E})$ is determined, the above equations then relate the nuclear structure theories to very extensive $\left(\pi, \pi^{\prime}\right)$ data.

Since $\tau(E)$ is a many-body operator, the calculation based on the above multiple scattering formulation is still very difficult. Most of the nuclear structure studies using $\left(\pi, \pi^{\prime}\right)$ were carried out by using a simplified DWIA in which $\tau(E)$ is replaced by $t(E)$ of the free $\pi N$ scattering in evaluating the transition potential Eq. (5). The optical potential $U_{00}$ is either deduced [4] from the $\Delta$-hole model or simply taken to be a phenomenological model [5] which is adjusted to fit the pion-nucleus elastic scattering differential cross sections. Since the free $\pi \mathrm{N}$ scattering amplitude $t(E)$ is a one-body operator, the calculation of the pion-nucleus transition potential $\mathrm{U}_{\mathrm{fO}}$ is greatly simplified. The DWIA amplitude, Eq. (4), then takes the following form

$$
T_{f 0}=\int d \vec{k} \int d \vec{k}^{\prime} \chi_{\vec{k}_{f}}^{(-) *}\left(\vec{k}^{\prime}\right)\left(t(\pi n) F_{f 0}^{n}(\vec{q})+t(\pi p) F_{f 0}^{p}(\vec{q})\right) \chi_{\vec{k}_{0}}^{(+)}(\vec{k})
$$

where $\vec{q}=\vec{k}-\vec{k}^{\prime} . \quad F_{f 0}^{(n, p)}(\vec{q})$ are the neutron and proton transition form factors which can be calculated from a given nuclear model. For example, its relation with the nuclear shell model was given explicitly in Ref. [6].

The important point to note is that in the $\Delta$ excitation region, the $\pi \mathrm{N}$ interaction has a very strong isospin-dependence: $t\left(\pi^{+} p\right) \sim 3 t\left(\pi^{+} n\right)$ and $t\left(\pi^{-} n\right) \sim 3 t\left(\pi^{-} p\right)$. Consequently, we have a very interesting ratio between $\left(\pi^{-}, \pi^{-1}\right)$ and $\left(\pi^{+}, \pi^{+}\right)$inelastic scattering cross sections

$$
R\left(\pi^{-} / \pi^{+}\right)=\frac{\sigma\left(\pi^{-}\right)}{\sigma\left(\pi^{+}\right)} \sim \frac{\left|F^{p}+3 \times F^{n}\right|^{2}}{\left|3 \times F^{p}+F^{n}\right|^{2}} .
$$

This formula was first verified [7] in the study of ${ }^{13} \mathrm{C}\left(\pi, \pi^{\prime}\right){ }^{13} \mathrm{C}\left(\frac{9^{+}}{2}, 9.5 \mathrm{MeV}\right)$. It was known that $\left(\frac{9^{+}}{2}, 9.5 \mathrm{MeV}\right)$ state can be formed by coupling a $\mathrm{d}_{5 / 2}$ neutron to a ${ }^{12} \mathrm{C}\left(2^{+}\right)$core. The transition from the ground state to this excited state only involves the neutrons, and hence $\mathrm{FP}=$ 0 in Eq. (7). This led to the prediction of $\mathrm{R}\left(\pi^{-} / \pi^{+}\right) \sim 9$ which was in good agreement with the data. Another interesting case is the ${ }^{18} \mathrm{O}\left(\pi, \pi^{\prime}\right)^{18} \mathrm{O}\left(2_{1}^{+}\right)$. If there is no excitation of the 160 core, one would expect $\mathrm{R}\left(\pi^{-} / \pi^{+}\right) \sim 9$. Experimentally, one finds $\mathrm{R}\left(\pi^{-} / \pi^{+}\right) \sim 2$. This implies that the polarization of the ${ }^{16} \mathrm{O}$ core is involved in the excitation mechanism. By using Eq. (7), one finds that $\mathrm{FP} / \mathrm{F}^{\mathrm{n}} \sim 0.5$ which is close to the prediction of a well-studied coexistence model of $18 \mathrm{O}$ [8]. The strong isospin-dependence, as illustrated in the above two cases, is the main advantage of using pions to probe the structure of nuclei. Extensive work on this aspect of pion-nucleus scattering can be found in the literature. The most relevant to many talks at this conference is the use of $\mathrm{R}\left(\pi^{-} / \pi^{+}\right)$to examine the relative importance between the neutron and proton excitations within the IBM-II of nuclear collective excitations. The prediction made in 1979 was later verified experimentally.

Although $\left(\pi, \pi^{\prime}\right)$ has been very useful in nuclear structure studies, it has an intrinsic deficiency. Because of the strong absorption of pions by nuclei, $\left(\pi, \pi^{\prime}\right)$ is sensitive mainly to the properties of nuclear surface. A more effective way is to replace the incident pion by a photon and to consider $(\gamma, \pi)$ and (e,e' $\pi)$ reactions. The experimental studies of these reactions 
are still limited so far, but can be carried out very efficiently at several new electron facilities in the near
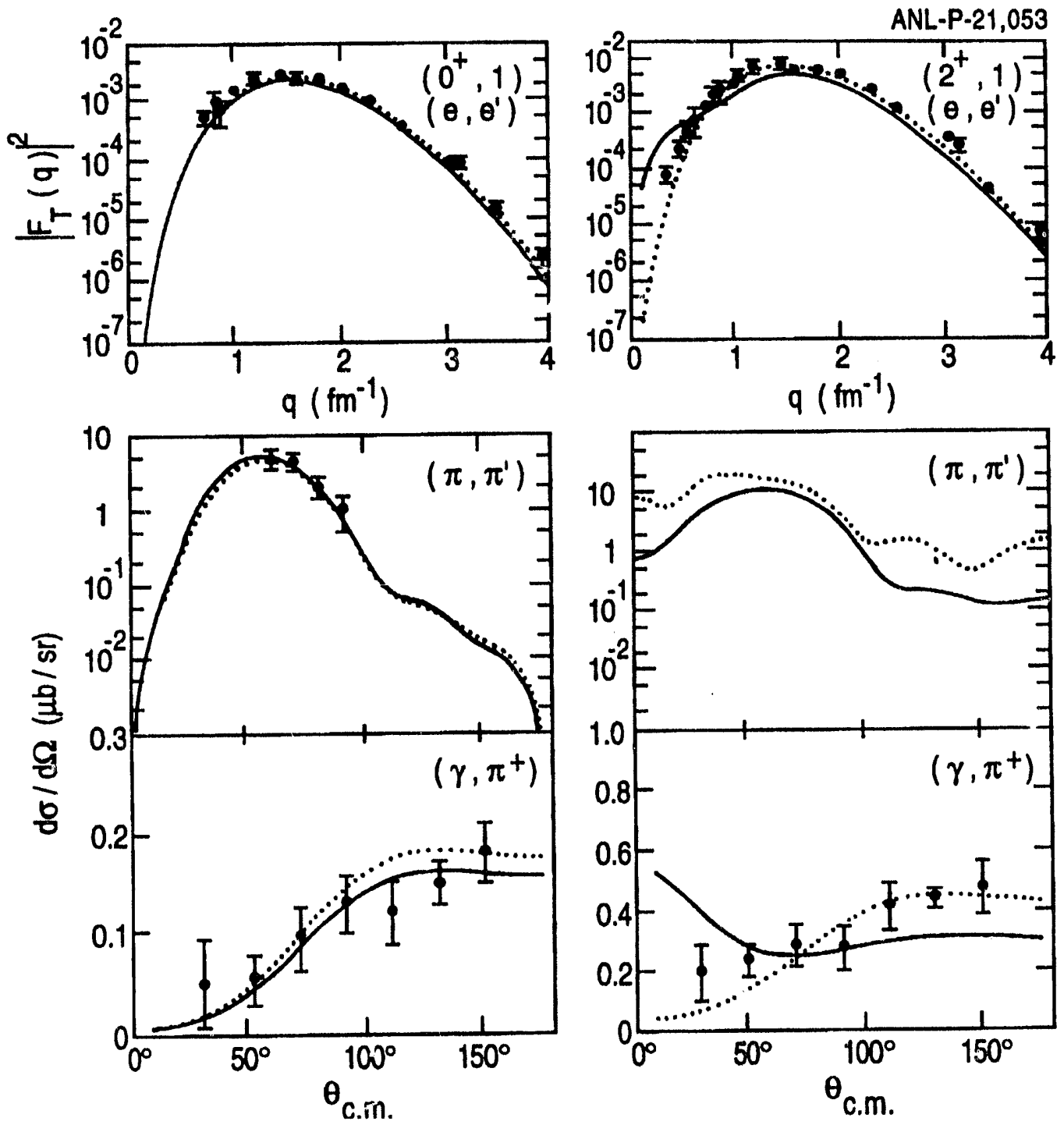

Figure 1

future. To motivate this development, we [9], in a collaboration between Argonne and Osaka University, have made extensive predictions of $(\gamma, \pi)$ reactions on 1 p-shell nuclei. The calculations have been done in DWIA based on the $\gamma \mathrm{N} \rightarrow \pi \mathrm{N}$ model of Nozawa, Blankleider and Lee [10]. The main feature of our approach is to account for the core polarization in a perturbation calculation including excitations up to $6 \hbar \omega$ and to treat the off-shell $\pi \mathrm{N}$ dynamics rigorously by performing calculations in momentum-space. In addition, we address various nuclear structure issues by examining whether a single nuclear model can simultaneously describe the data of $\left(e, e^{\prime}\right),\left(\pi, \pi^{\prime}\right)$, and $(\gamma, \pi)$ reactions. In Figs. 1 and 2 we show some of our results. Figure 1 shows the difference between the predictions from the shell model of Cohen and Kurath [11] (solid curves) and the shell model of Hague and Maripuu [12] (dotted curves). Their differences are clearly distinguishable in the case of $\left(2^{+}, 1\right)$ at $5.7 \mathrm{MeV}$. In Fig. 2, we 
show that core polarization is essential in obtaining agreement with the data of $\left(e, e^{\prime}\right)$ and $\left(\pi, \pi^{\prime}\right)$ reactions leading to the $\left(4^{+} 0\right)$ state at $6.025 \mathrm{MeV}$. The solid curves are obtained when the core polarization effects are included, while the dotted curves are the predictions of the model of Cohen and Kurath. In the same figure, we also see the deficiency of the model of Cohen and Kurath in describing the very weak transition to the $\left(3^{+}, 0\right)$ state at $4.77 \mathrm{MeV}$ which has the same spin and isospin as the ${ }^{10} \mathrm{~B}$ ground state. It is pointed out by Kurath [13] that with only a $1 \%$ admixture of these two states, the predicted $\left(\pi, \pi^{\prime}\right)$ cross section, which is dominated by the $\mathrm{L}=2$ and $\mathrm{S}=0$ transition, can be reduced to the experimental value while the changes in the predicted magnetic moment and quadruple moment of the ${ }^{10} \mathrm{~B}$ ground state are negligible. Experimental verification of the predictions for the $\left(\gamma, \pi^{0}\right)$ reaction presented in Fig. 2 will be very interesting.
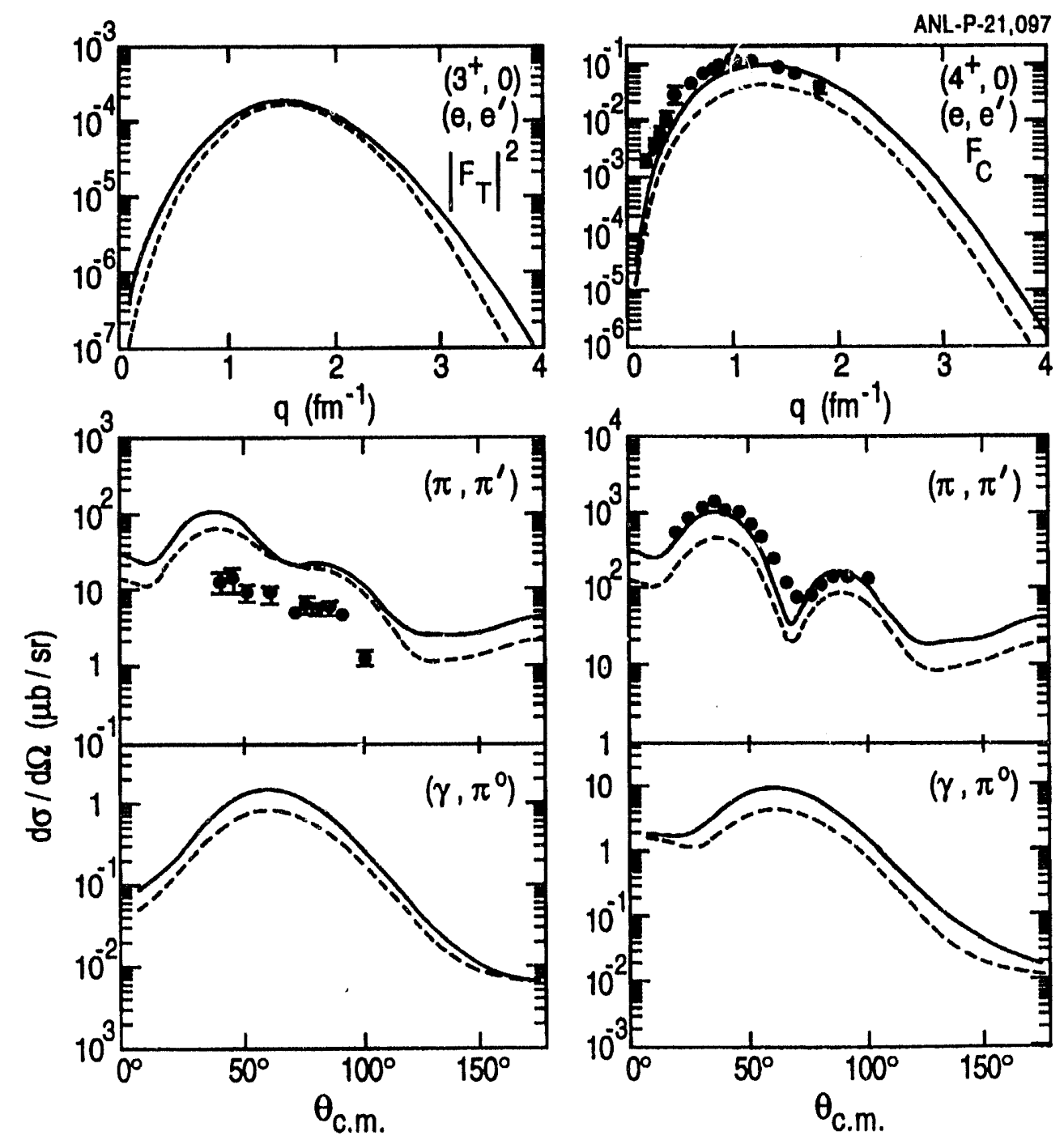

Figure 2 
The pion can undergo a double-charge-exchange (DCX) scattering on a pair of nucleons inside the nucleus. The DCX can therefore probe the nucleon-nucleon correlations. As an example, we mention that a recent study [14] of ${ }^{11} \mathrm{~B}\left(\pi^{-}, \pi^{+}\right)^{11} \mathrm{Li}$ proves to be unique in probing the neutron halo of ${ }^{11} \mathrm{Li}$ which was first observed in the $\left({ }^{11} \mathrm{Li},{ }^{2} \mathrm{Li}\right)$ fragmentation reaction of heavy nuclei. By using the three-body model of Bertsch and Esbensen [15] to describe the ground state of ${ }^{11} \mathrm{Li}$ as an $s$-wave nn-pair moving in a mean field of an inert ${ }^{9} \mathrm{Li}$ core, the DWIA calculation of the ${ }^{11} \mathrm{~B}\left(\pi^{-}, \pi^{+}\right)^{11} \mathrm{Li}$ reaction agrees with the data very well. This is shown in Fig. 3 in which the contributions from the $\mathrm{J}=0$ and $\mathrm{J}=2$ components of the nn pair are also displayed.

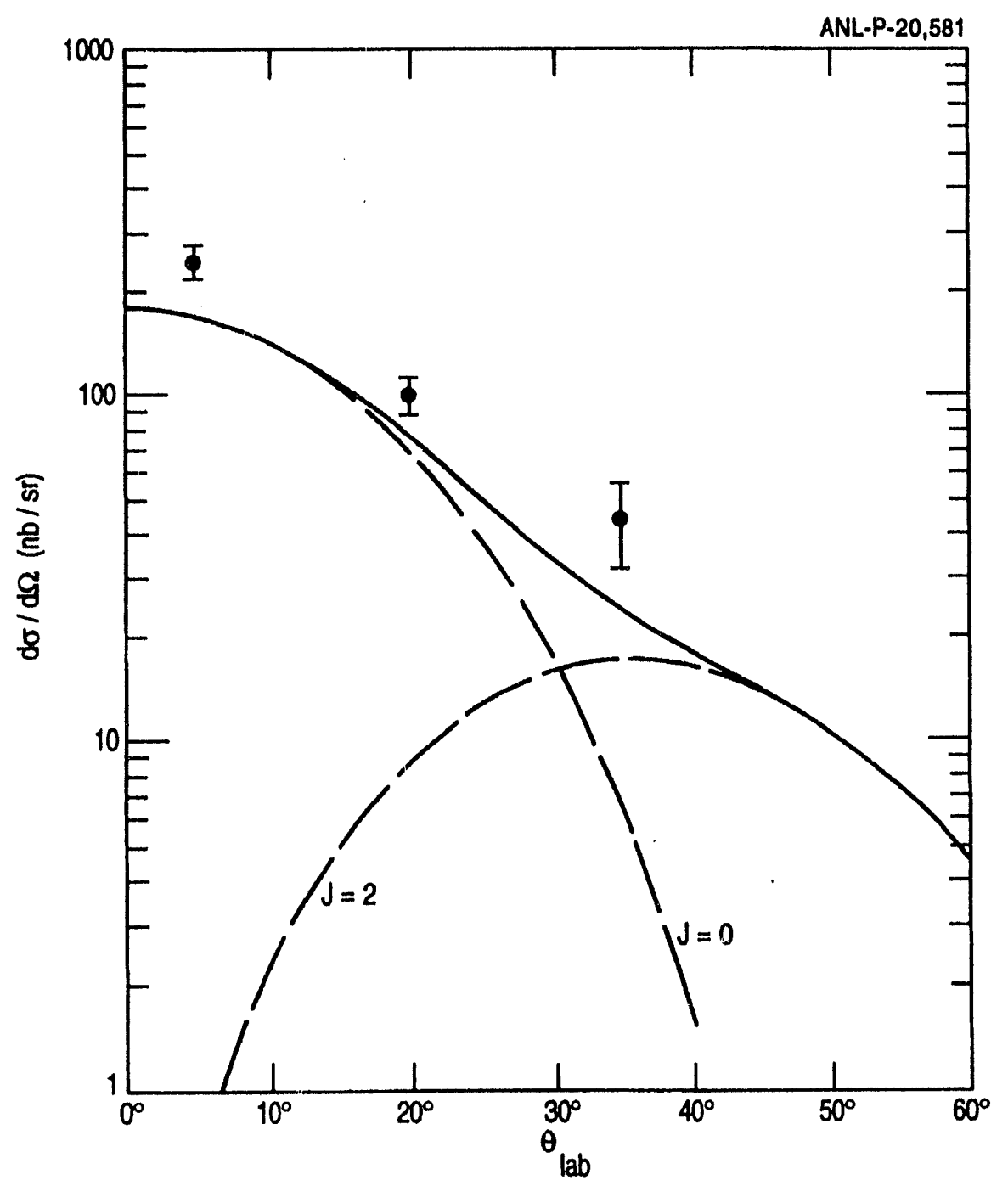

Figure 3

Extensive DCX measurements have been performed for nuclei throughout the periodic table. While some general features, such as the A-dependence, can be understood qualitatively, the DCX cross sections predicted by DWIA or coupled-channels approaches within the context of nuclear shell model are often not in agreement with the data. It is necessary to develop a 
more detailed understanding of the DCX reaction mechanism and an approach more sophisticated than the nuclear shell model to describe nuclear correlations. Similar difficulties are also encountered in the study of pion absorption process $(\pi, N N)$ which also involves twonucleon in the basic reaction mechanism. The lack of a satisfactory description of DCX and pion absorption is a very unsatisfactory aspect of the pion physics. A useful next step is to develop a theoretical framework in which the DCX and $(\pi, N N)$ reactions can be related to the $(\gamma, \mathrm{NN})$ and $\left(\mathrm{e}, \mathrm{e}^{\prime} \mathrm{NN}\right)$ reactions which are also sensitive to the nuclear correlations, but do not have the complications due to the initial pion distortions. This is an exciting new direction, since accurate data of $(\gamma, \mathrm{NN})$ and $\left(e, e^{\prime} \mathrm{NN}\right)$ reactions will become available at NIKHEF, Mainz and CEBAF. The most fruitful measurements would be for the cases to which the corresponding DCX and pion absorption data already exist.

In addition to carrying out the $(\gamma, \pi)$ and $(\gamma, N N)$ studies as suggested above, there will be new opportunities at CEBAF. Armed with GeV continuous electron beams, it is possible to obtain accurate data in the kinematic region where the higher mass nucleon resonances $\mathrm{N}^{*}$ can be excited. These resonances have a large decay width for decay into $\pi \Delta, \rho N, \eta N$, and $\mathrm{K} \Lambda$ states. To proceed, it is necessary to first develop an appropriate theoretical formulation of the problem. It is doubtful that the simple DWIA will be appropriate because of the presence of two-pion channels in the $\mathrm{N}^{*}$ decay. The extension of the $\Delta$-hole model to $\mathrm{N}^{*}$-hole model is also questionable, because, unlike the $\Delta$ in the $P_{33}$ channel, the non-resonant interaction is comparable to, if not larger than, the resonant $\mathrm{N}^{*}$ mechanism.

In summary, in the past two decades much nuclear structure information was obtained by using pion as a probe. The difficulties encountered in describing the DCX and pion absorption processes clearly indicate the need of combining these studies with the studies using electromagnetic probes. This will be the focus of the research when CEBAF starts operation in 1994.

\section{ACKNOWLEDGMENTS}

This work is supported by the U.S. Department of Energy, Nuclear Physics Division, under contract W-31-109-ENG-38.

\section{REFERENCES}

[1] M. Hirata, J. H. Koch, F. Lenz, and E. J. Moniz, Ann. Phys. (NY) 120, 205 (1979);

Y. Horikawa, M. Thies, and F. Lenz, Nucl. Phys. A345, 386 (1980);

E. Oset and W. Weise, Nucl. Phys, 329, 365 (1979).

[2] T.-S. H. Lee and K. Ohta, Phys. Rev. C 25, 3043 (1982).

[3] A. K. Kerman, H. McManus, and R. Thaler, Ann. Phys. (NY) 8, 551 (1959).

[4] B. Karaoglu and E. J. Moniz, Phys. Rev. C 33, 974 (1986).

[5] J. A. Carr, H. McManus, and K. Striker-Bauer, Phys. Rev. C 22, 2043 (1980).

[6] T.-S. H. Lee and D. Kurath, Phys. Rev. C 21, 293 (1980).

[7] T.-S. H. Lee and D. Kurath, Phys. Rev. C 22, 1670 (1980).

[8] T.-S. H. Lee and R. D. Lawson, Phys. Rev. C 21, 679 (1980).

[9] T. Sato, N. Odagawa, H. Ohtsubo, and T.-S. H. Lee, ANL preprint (1993).

[10] S. Nozawa, B. Blankleider, and T.-S. H. Lee, Nucl. Phys. A513, 459 (1990).

[11] S. Cohen and D. Kurath, Nucl. Phys. 73, 1 (1965).

[12] P. S. Hauge and S. Maripuu, Phys. Rev. C 8, 1609 (1973).

[13] D. Kurath, private communication, 1993.

[14] H. Esbensen, D. Kurath, and T.-S. H. Lee, Phys. Lett. B287, 289 (1993).

[15] G. F. Bertsch and H. Esbensen, Ann. Phys. 209, 327 (1991). 

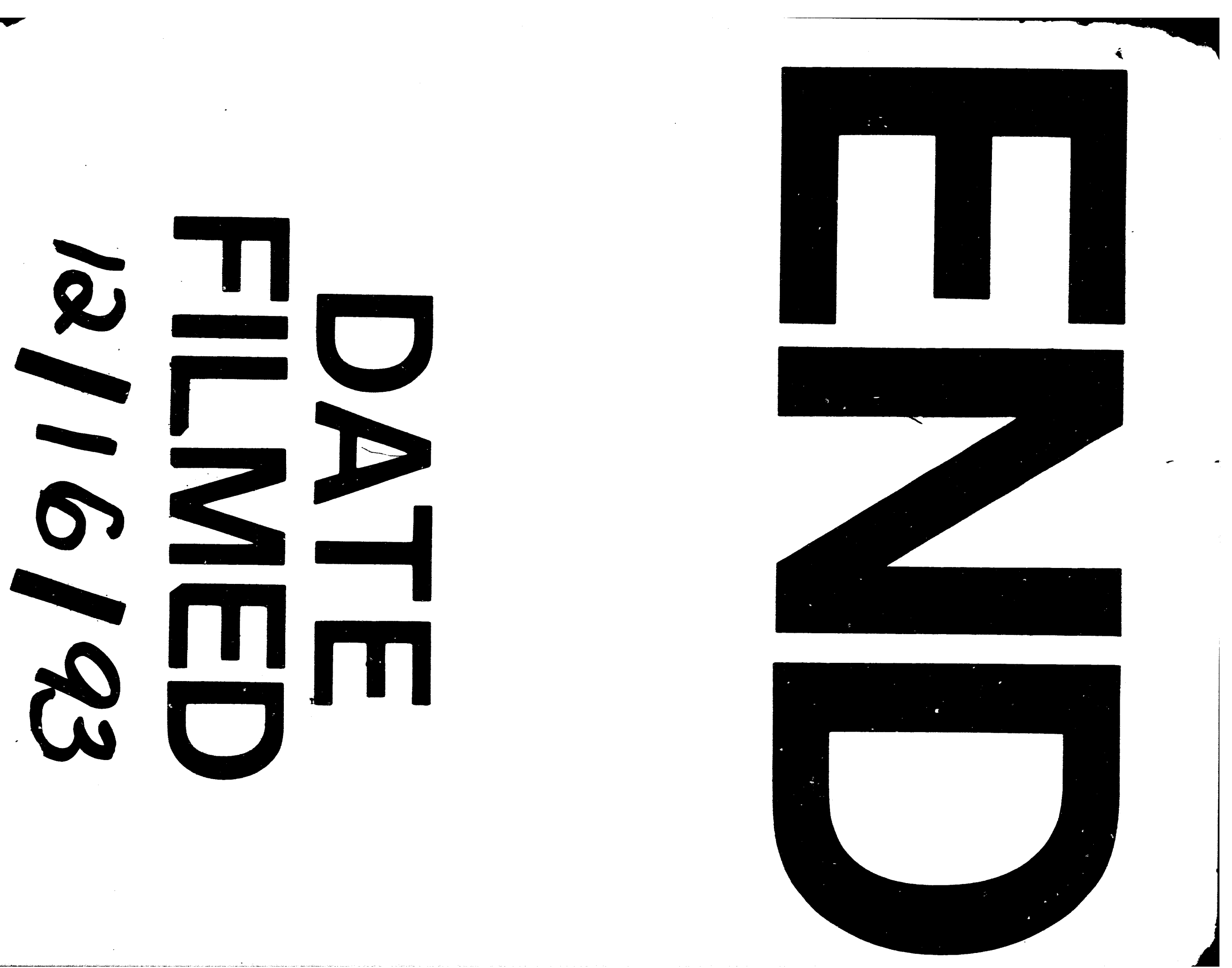
